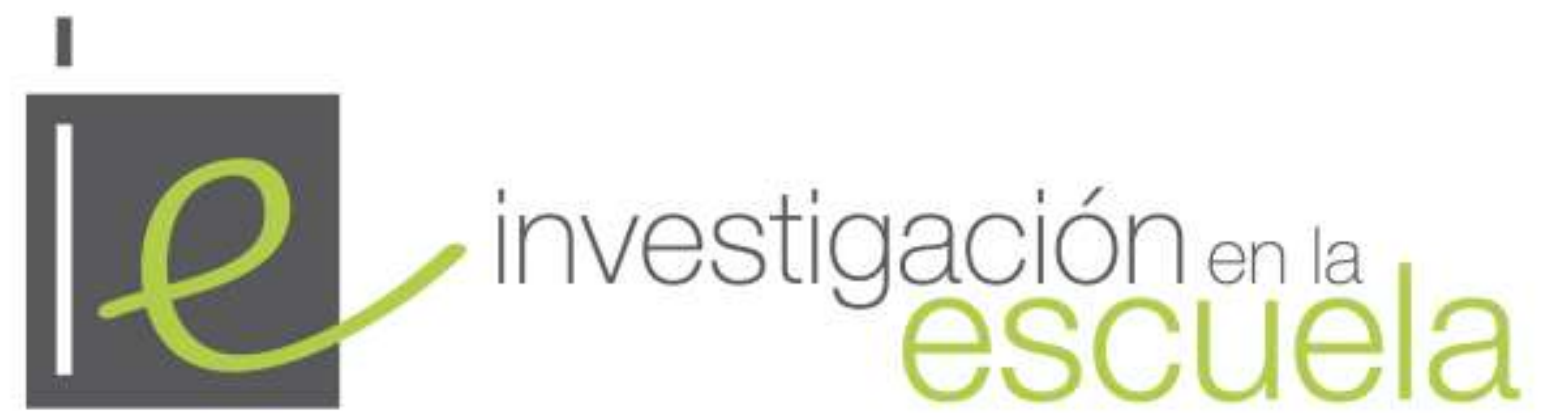

Revista internacional de investigación e innovación educativa

\title{
Características de la función docente en centros públicos no universitarios. Las dificultades del desempeño laboral de la docencia en la ciudad de Melilla y estrategias de mejora \\ Irene Orellana Román \\ Universidad de Granada \\ España
}

Citación: Orellana Román, I. (2018). Características de la función docente en centros públicos no universitarios. Las dificultades del desempeño laboral de la docencia en la ciudad de Melilla y estrategias de mejora. Investigación en la Escuela, 95, 43-62. Recuperado de: http://www.investigacionenlaescuela.es/articulos/R95/R95-4

Resumen: La formación académica a la que se somete el docente influye en su desarrollo profesional y de ello dependerá el desempeño de su función diaria y la resolución de los problemas con los que se enfrenta en el aula. La discusión se encuentra en la divergencia entre dicha preparación y la realidad laboral a la que se enfrentan. Este trabajo tiene como objetivo recoger información sobre las características del desarrollo profesional docente a través de las opiniones de los mismos. Para ello se recogió la opinión de 160 docentes de la Ciudad Autónoma de Melilla sobre los principales aspectos que influyen en su trabajo y la formación necesaria para afrontarlos. Como instrumento de recogida de datos se utilizó el cuestionario cedido por Cardona y se aplicó a 160 docentes melillenses de niveles no universitarios. Los resultados obtenidos muestran diferencias significativas entre los distintos niveles educativos y los años de experiencia del profesorado, lo que evidencia que tanto los problemas a los que se enfrenta el profesorado como las estrategias que utilizan para disuadir dichos problemas, son diferentes según el nivel educativo y los años de experiencia. La conclusión a la que se llega es el necesario cambio en la formación del docente conforme a la realidad de las aulas. 
Palabras clave: "Formación docente"; "iniciación a la docencia"; "profesor novel”; "profesor experimentado"; "problemática docente"; "estrategia de mejora".

Characteristics of the teaching function in public non-university centers Subtitle: The difficulties of the work performance of teaching in the city of Melilla and improvement strategies

Abstract: Academic training that a teacher undergoes influences his professional development and his daily function and his skill on problem solving will depend on it. The discussion diverges between that preparation and the labor activity to which a teacher deals with. This work (project) has as main aim to collect information about teacher training and their professional development through the opinion of the subjects themselves. In order to do that 160 opinions of Autonomous City of Melilla teachers about the main aspects that affect their work and the required training to affront then were collected. The questionnaire given by Cardona was used as an instrument of date collection and it was applied to 160 teachers from Melilla which didn't have a degree level. The results show significant differences between the different educational levels and years of teaching experience, which shows that, on the one hand, depending on the educational stage in which they teach, the problems that teachers have to face varies. On the other hand, the chosen strategies to minimize or eliminate the problems of teacher performance alter according to the years of experience of teacher. The conclusion reached is that a necessary change in teacher formation so that it resembles the reality of school.

Key words: "Teacher training"; "initiation to teaching"; "new teacher"; "experienced teacher"; "teaching problems"; "improvement strategy".

Caractéristiques de la fonction d'enseignement dans les centres publics non universitaires Sous-titre: Les difficultés de l'exécution du travail de l'enseignement dans la ville de Melilla et les stratégies d'amélioration

Resumè: La formation académique aux enseignants influence subit leur développement professionnel et cela dépendra de la performance de leur fonction quotidienne et la résolution des problèmes auxquels elle est confrontée dans la salle de classe. La discussion est la divergence entre cette préparation et la réalité du travail qu'ils font face. Ce travail a pour principal objectif de recueillir des informations sur la formation des enseignants et le perfectionnement professionnel d'entre eux par les opinions des sujets eux-mêmes. Pour ce faire, l'opinion de 160 enseignants dans la ville autonome de Melilla sur les principaux aspects qui influent sur leur travail et la formation nécessaires pour y répondre recueillies. Comme questionnaire de l'instrument de collecte de données fournies par Cardona et appliquée à 160 niveaux universitaires melillenses enseignants utilisés. Les résultats montrent des différences significatives entre les différents niveaux d'enseignement et des années d'expérience des enseignants, ce qui montre que les problèmes que les enseignants sont confrontés et les stratégies qu'ils utilisent pour dissuader ces problèmes sont basés sur le niveau d'instruction et années d'expérience. La conclusion est le changement nécessaire dans la formation des enseignants en fonction de la réalité de la classe.

Mots-clés: "Formation des enseignants"; "initiation à l'enseignement"; "nouvel enseignant"; "enseignant expérimenté"; "problèmes d'enseignement"; "stratégie d'amélioration".

\section{Introducción}

La investigación sobre el profesorado es uno de los tópicos más investigados en el campo de la investigación educativa (Cardona, 2008; Imbernón, 1998; Infante, 2010; Veenman, 1984). Uno de 
los principales objetivos que debe cumplir la investigación educativa es el de responder a las demandas y necesidades de la sociedad, y la formación del docente pasa por juicios de valor debidos a la transformación de la sociedad en general y al cambio del alumnado en particular.

Para permitir la mejora del sistema educativo, se necesitan conocer las dificultades y características de la labor docente, objeto del presente estudio.

\section{Fundamentación teórica}

\section{La formación del docente y la realidad educativa}

A principios del siglo XXI aumentó el interés por el papel del profesorado en el sistema educativo. Aspectos como la preparación inicial, el desarrollo profesional, las condiciones de trabajo y su papel en la comunidad educativa son analizados. (Tiana, 2013).

Existen discrepancias debidas a que la formación inicial de nuestro profesorado es estática, caduca, demasiado teórica y desprovista de unión entre la universidad y los centros educativos. (Feliz, 2008) (citado en Feliz y Ricoy, 2008, p. 8). Estas discrepancias entre formación inicial y realidad educativa pueden observarse en diferentes estudios, como el de Rico (2006) y el de Epelde (2012) acerca de las limitaciones estratégicas con las que se enfrentan los estudiantes de Educación Primaria en sus periodos de prácticas. En estos estudios se pone de manifiesto que los futuros maestros y maestras no poseen la formación suficiente en la organización del alumnado, en el control y en la disciplina en el aula, en mantener la atención de los y las discentes y en hacer uso de recursos didácticos convenientes, entre otros aspectos.

Si ponemos la vista en los profesionales de secundaria, esta problemática se agrava, ya que su única formación pedagógica es o el ya derogado Certificado de Aptitud Pedagógica (CAP), de tres meses de duración, o el actual Máster Universitario de Formación del Profesorado, de un curso académico de duración.

En otro estudio relacionado con estudiantes de este máster en fases de prácticas, estos establecen aprender más sobre la profesión docente de Secundaria en sus periodos de prácticas que en la fase teórica del máster (Rodríguez y Torrego, 2013), lo que recalca la disociación entre la formación recibida en la universidad y la realidad laboral que se encuentran los futuros docentes. Otra investigación previa a esta, diseñada dese la Universidad de Zaragoza, resaltaba la necesidad formativa en Tecnologías de la Información y la Comunicación y en innovación curricular para el alumnado de prácticas del máster de Secundaria. (Ramírez, Sánchez, García, y Latorre, 2011; Valverde, Garrido, y Fernández, 2010).

Como puede observarse, la controversia entre formación teórica y realidad educativa es un hecho que se ve acrecentando en los docentes nuevos, siendo estos los que tienen menos de cinco años de experiencia (Cardona, 2008). Se muestran inexpertos y desprovistos de estrategias para mitigar con el día a día de las aulas. De hecho, algunos autores llaman "Fase de supervivencia y exploración” a este período (González, 1999) debido al elevado número de problemas por los que atraviesan.

De estos y otros estudios, se convierte en explícita la necesidad de mejorar la formación inicial docente, ya sea de Primaria o Secundaria. Esta mejora redundará en la calidad educativa ya que, tal y como afirma Tiana (2013) a partir del Informe How the world's best-performing school systems come out on top (McKinsey \& Company, 2007), la calidad del profesorado es el factor de mayor importancia en los resultados académicos de los discentes.

Si la calidad educativa depende de la calidad docente, teniendo en cuenta que la competencia docente es el conjunto de habilidades, actitudes, aptitudes, percepciones y capacidades que se aplican en el contexto educativo (Osorio, Herrer y Quiles, 2016), un contexto educativo que evoluciona 
debe transformarse casi a la vez que esta formación. Por tanto, la formación docente debe estructurarse de acuerdo a las necesidades del aula, a las características del alumnado y a los hitos que definen la sociedad en la que se encuentra la comunidad educativa, ya que "las estrategias eficaces son aquellas que se plantean desde el centro en función de su realidad e implica a su profesorado para buscar respuestas educativas adecuadas a su problemática” (Arteaga y García, 2008, p. 254). Sería por tanto, la investigación-acción, la herramienta principal para la formación docente, tal y como establecen algunos autores como Anderson (2010) (citado en Pérez, 2016, p.49), afirman que los mejores profesores son aquellos que continúan aprendiendo y actualizando sus estrategias docentes.

\section{Fundamentos y características de la formación}

Con todos los problemas y dificultades que encuentran los docentes para el desempeño de su función laboral (Diker y Terigi, 1997; Vázquez, Sarramona y Touriñan, 2010; Veenam, 1984), parece claro que estamos ante un "problema de calidad de la formación inicial" (Márquez, 2009:40).

Esta calidad formativa inicial puede superarse si, según Tiana (2013), para ello se dispone de los mejores docentes a través de las siguientes estrategias:

1) Seleccionar a los mejores estudiantes para que sean maestros;

2) Pagarles un buen salario inicial como docentes.

Estas acciones fomentan el aumento del estatus de los estudios de profesorado al hacer más exigente el acceso y, además, permite utilizar mejor los recursos disponibles al disminuir el número de estudiantes.

Existen dos modelos de formación inicial del profesorado: el modelo simultáneo y el modelo consecutivo. En el primero de ellos, la formación académica y didáctica se integra en un mismo plan de estudios, como son los estudios de Educación Infantil o Educación Primaria. En el modelo consecutivo, primeramente se adquiere una formación académica de especialización en un plan de estudio (Grados EEES) y, posteriormente, se realiza la formación pedagógica necesaria para la profesión docente, lo que se conoce como el Máster de Secundaria (Rebolledo, 2015).

Según Mérida (2009) (citado en Rebolledo, 2015:131), son las instituciones educativas las que tienen la responsabilidad de mejorar la formación inicial, ya que tienen el poder de establecer la realidad de las tareas educativas necesarias para cumplir con el desempeño docente.

Por su parte, Feliz y Ricoy (2008) establecen cuatro focos principales en la formación inicial del profesorado: la formación cultural general, la humana, la científica y disciplinar, y la técnica. Y Tiana (2013) recomienda el establecimiento de tutorías para apoyar al profesorado en su trabajo docente, la formación de líderes instructivos eficaces y la oferta de posibilidades para que los profesores puedan aprender de otros docentes.

Siguiendo las ideas de Jordán (2007), (citado en López-Gómez y Pérez-Navío, 2013, p. 35.), la formación permanente resulta más importante ya que la preparación inicial es escasa. Al igual que se promulga la formación inicial basada en la realidad educativa, la formación permanente debe construirse de la misma forma. En este caso, la investigación-acción, caracterizada por centrarse en los problemas de preocupación inmediata, por buscar soluciones prácticas y por estimular la colaboración de los participantes en igualdad (Moral, 2000), es la opción formativa más adecuada.

Esta colaboración que caracteriza a la investigación-acción, se concibe como la integración del desarrollo de procesos cognitivos del profesorado, la indagación científica y la formación centrada en la escuela, que implica un estilo abierto y práctico entre los miembros de la comunidad educativa (Medina, 2015).

Como se aboga por el protagonismo del profesor en su formación, es necesario conocer la opinión que tienen éstos sobre su preparación formativa, el desarrollo de su actividad profesional y las características de su desempeño docente. 


\section{Objetivos}

El objetivo principal de esta investigación es recoger información acerca de la problemática de la función docente en niveles no universitarios en Melilla. Los objetivos específicos de este estudio fueron los siguientes:

- Averiguar cuáles son los aspectos que influyen en el desempeño de la función docente.

- Determinar cuáles son las estrategias para mejorar el desempeño de la función docente.

- Conocer los rasgos del profesorado principiante.

- Identificar las estrategias para la iniciación del profesorado novel.

- Seleccionar las medidas para evitar o disminuir los problemas del profesorado principiante y del experto.

\section{Descripción del contexto}

Esta investigación se realiza en la ciudad española de Melilla, de $13.41 \mathrm{~km} 2$ y una población total de 83679 habitantes.

Su situación geográfica y antecedentes históricos le dan a Melilla unos rasgos culturales peculiares, entre los que destacan la coexistencia de colectivos marcadamente diferenciados en cuanto a lengua, tradiciones, cultura y religión. Los dos colectivos mayoritarios son el de origen hispano, identificado con las costumbres españolas y la religión católica, y el de origen rifeño o bereber, con lengua materna el Tamazight y con religión islámica. Existen también otros colectivos minoritarios como el hebreo, el hindú y la etnia gitana.

Al mismo tiempo, cohabitan un elevado número de personas de origen marroquí, y se desconoce el número de personas en situación ilegal, además de las personas que se encuentran en los Centros de Estancia Temporal de Inmigrantes y Centro de Menores No Acompañados.

La ciudad se divide en ocho distritos, siendo algunos denominados por el MECD como "centros de difícil desempeño" (SATE, 2013), de los que solo dos participan en el estudio. Las principales características de estos centros son la falta de motivación del alumnado, las necesidades educativas, la pertenencia a familias en riesgos de exclusión social, el bajo nivel socio-económico y la alta tasa de paro en las familias.

\section{Diseño del proceso de investigación}

\section{Método}

Este estudio de naturaleza cuantitativa se presenta como una investigación no experimental, transversal y descriptiva, cuyo instrumento principal de recogida de datos es un cuestionario individual, analizado con el programa SPSS.

El procedimiento seguido para la realización de esta investigación ha sido el siguiente:

- Presentación de la investigación a través de entrevistas con el equipo directivo a todos los centros públicos de la ciudad.

- Acceso a la muestra y aplicación del cuestionario, previa autorización de la Dirección Provincial y con el consentimiento de la Dirección de cada centro educativo.

- Clasificación y codificación de datos en el SPSS 20.0.

- Interpretación de los datos. 
Las técnicas de análisis de datos que se han llevado a cabo, una vez se ha analizado la validez de la escala y el margen de error de la muestra, ha sido, en primer lugar, un análisis descriptivo de los datos utilizando la prueba. Se han tenido en cuenta como variables categóricas las siguientes:

- El nivel educativo en el que imparten docencia:

- Infantil.

- Primaria.

- ESO y Bachillerato.

- Formación Profesional.

- La edad:

- De 24 a 35 años.

- De 35 a 46 años.

- De 46 a 57.

- De 57 a 68.

- Los años de experiencia docente.

- Menos de 5 años (noveles).

- De 5 a 10 años.

- De 10 a 15 años.

- De 15 a 20 años.

- $\quad$ Más de 20 años.

En segundo lugar, se ha llevado a cabo un análisis relacional de las anteriores variables con cada una de las dimensiones del cuestionario.

La categoría $(\mathrm{K})$ establecida con la variable edad, donde se establecen 4 categorías, se debe a que al seguir la Regla de Sturges, el número de categorías resultante es 9, careciendo de significatividad para el estudio. Para la claridad del mismo, se decide establecer que $K=4$ con una amplitud (A) de 11, según la fórmula $A=\frac{R}{K}$. Las categorías en los años de experiencia se deben a razones teóricas (Cardona, 2008; Castejón, 2010; Rojas, Carrillo y Flores, 2012), por las cuales un docente es novel con menos de 5 años de experiencia. Para igualar en amplitud los grupos de edad de la muestra, se decide que A es 5 , y K es 5 .

\section{Descripción del instrumento}

El instrumento utilizado para la recogida de datos fue un cuestionario cerrado de valoración mediante una escala tipo Likert de cinco puntos, donde 1 es poco relevante, y 5 muy relevante. Se denomina "Estudio sobre la problemática del profesorado en su función docente" (Cardona, 2008) y se compone de 4 partes: presentación y descripción del estudio; datos de identificación; observaciones; y cuerpo del cuestionario con 83 ítems divididos en 6 dimensiones:

1. Aspectos que pueden interferir en el desempeño de la función docente ( 25 ítems).

2. Rasgos que suelen otorgarse al profesorado principiante (7 ítems).

3. Estrategias de iniciación a la docencia (15 ítems).

4. Medidas que pueden evitar o disminuir los problemas del docente novel (14 ítems).

5. Medidas que pueden evitar o disminuir los problemas del docente con experiencia (12 ítems).

6. Medidas para mejorar el desempeño de la función docente del profesorado (10 ítems). El cuestionario ya construido tiene una fiabilidad del $94.9 \%$, según indica la Tabla 1. La validez de la escala es aceptable ya que cada ítem tiene una correlación significativa con el criterio 
(Gil, 2011). El KMO es cercano a la unidad, 0,796 y la prueba de Barlett tiene un valor alto que puede considerarse significativo (Tabla 2).

Tabla 1

Estadísticos de fiabilidad

Alfa de Cronbach $\quad$ N de elementos

\begin{tabular}{ll}
\hline, 949 & 83 \\
\hline
\end{tabular}

Tabla 2

KMO y prueba de Bartlett

Medida de adecuación muestral de Kaiser-Meyer-Olkin. $\quad$,796

$\begin{array}{lll}\text { Prueba de esfericidad de Bartlett } & \text { Chi-cuadrado aproximado } & 9639,788\end{array}$

gl 3403

Sig. $\quad$, 000

\section{Descripción de la muestra}

Según los datos AVANCE, Melilla cuenta en el momento del estudio con un total de 1219 docentes en Enseñanzas de Régimen General. En un muestro no probabilísticos por conveniencia.

En este caso, la población la forman 1219 individuos, maestros y profesores de Infantil, Primaria, Secundaria y Formación Profesional de Melilla y han participado los sujetos que voluntariamente han estado interesados. El nivel de confianza va a ser del $95 \%$, por lo tanto z $=$ 1.96. Para un error del $5 \%$, se tiene que e $=0.05$. A falta de otros datos y para mayor seguridad se supone que $\mathrm{pq}=(0.50)(0.50)=0.25$.

Por lo tanto, y siguiendo la fórmula para poblaciones finitas, se obtiene que la muestra necesaria será de 292 sujetos. La muestra definitiva la componen 160 docentes, lo que representa el $13.12 \%$ de la población. El margen de error para la muestra real que he obtenido es del 7.22\%. La muestra tiene las siguientes notas características:

- El 68.1\% son mujeres, frente al 31.9\% de hombres, variable no tenida en cuenta para el análisis relacional del estudio, pues se ha considerado la igualdad entre hombres y mujeres y se ha evitado la identificación de diferencias por razón de sexo.

- La media de edad son 42,5 años.

- Teniendo en cuenta el nivel educativo en el que imparten enseñanza, el 10.6\% de los y las docentes de la muestra pertenecen a Educación Infantil, el $42.5 \%$ a Educación Primaria; el $34.4 \%$ a Secundaria y Bachillerato; y el $12.5 \%$ a Formación Profesional.

- La experiencia laboral media es de 15 años. 
Tabla 3

Grupo edad

\begin{tabular}{lcc}
\hline & Frecuencia & Porcentaje \\
\hline Menor de 35ños & 28 & 17,5 \\
Entre 35 y 46 años & 75 & 46,9 \\
Entre 46 y 57 años & 47 & 29,4 \\
Mayor de 57 años & 10 & 6,3 \\
Total & 160 & 100,0 \\
\hline
\end{tabular}

Tabla 4

Nivel educativo

\begin{tabular}{lcc}
\hline & Frecuencia & Porcentaje \\
\hline Infantil & 17 & 10,6 \\
Primaria & 68 & 42,5 \\
Secundaria y Bachillerato & 55 & 34,4 \\
Formación Profesional & 20 & 12,5 \\
Total & 160 & 100,0 \\
\hline
\end{tabular}

Tabla 5

Años de experiencia

Frecuencia

Porcentaje

Menos de 5 años

Entre 5 y 10 años

Entre 10 y 15 años

Entre 15 y 20 años

Más de 20 años de experiencia

Total
20

26

42

21

51

160
12,5

16,3

26,3

13,1

31,9

100,0 


\section{Resultados}

Los resultados obtenidos, teniendo en cuenta la totalidad de la muestra, han sido los siguientes:

Tabla 6

Puntuación de la Dimensión 1: problemas que afectan en el aula

Ítems

Media

A. Disciplina en el aula 3,98

B. Motivación de los alumnos 4,08

C. Adaptación de la enseñanza a las diferencias individuales.

D. Evaluar a los alumnos

E. Relaciones con los padres

F. Organización del trabajo en clase

G. Insuficiencia de material

H. Problemas con alumnos en concreto

I. Sobrecarga de trabajo

J. Relaciones con los otros profesores

2,77

K. Falta de tiempo para preparar las clases

L. El dominio de los diferentes métodos de enseñanza

M. Vigilancia de las normas de la escuela

N. Determinar el nivel de aprendizaje de los alumnos

O. Dominio de las materias

P. Trabajo burocrático

Q. Relaciones con el Equipo Directivo

R. Materiales didáctico inadecuados

S. Tratamiento de alumnos de aprendizaje lento

T. Tratamiento de la interculturalidad.

2,92

U. Dominio de los libros de texto y guías curriculares 2,68

V. Falta de tiempo libre

X. Falta de apoyo y orientación

Y. Número de alumnos por aula 


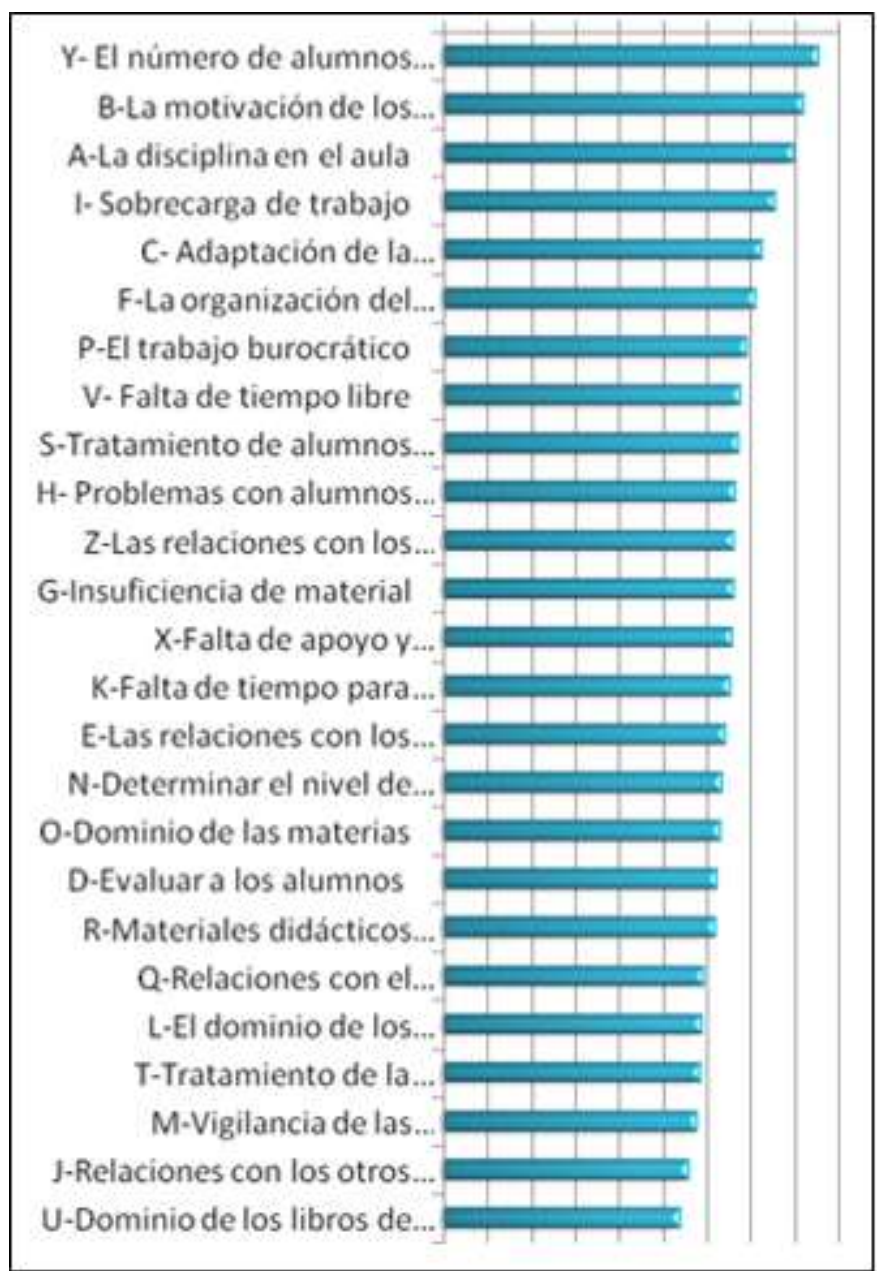

Figura 1. Dimensión 1.Problemas que de mayor a menor grado afectan al desarrollo docente en el aula y en el centro.

Tabla 7

Puntuación de la Dimensión 2: rasgos del profesorado principiante

\begin{tabular}{lc}
\hline Ítems & Media \\
\hline A2. Se encuentra entre dos identidades. & 3,06 \\
B2. El estrés, la angustia, diferentes miedos cuando no momentos de verdadero pánico. & $\mathbf{3 , 2 5}$ \\
C2. Necesita mucha más energía, tiempo y concentración para resolverlos problemas. & $\mathbf{3 , 5 9}$ \\
D2. Su gestión del tiempo no es muy segura, lo que provoca fatiga y tensión. & 2,79 \\
E2. Se encuentra en un estado de sobrecarga cognitiva. & 2,65 \\
F2. Se siente bastante solo, ya que ha perdido el contacto con sus compañeros de & 2,38 \\
estudios y todavía no se siente bien integrado con sus nuevos colegas. & \\
G2. No se distancia lo suficiente entre su papel y las situaciones problemáticas. & 2,53 \\
\hline
\end{tabular}


docencia en la ciudad de Melilla y estrategias de mejora

Tabla 8

Puntuación de la Dimensión 3: estrategias para iniciar al profesorado principiante

\begin{tabular}{lc}
\hline Ítems & Media \\
\hline A3. Materiales escritos sobre condiciones de trabajo y normas de la escuela. & 3,43 \\
B3. Reuniones y visitas previas al colegio. & 3,48 \\
C3. Seminarios sobre curriculum y enseñanza eficaz. & 3,29 \\
D3. Sesiones de entrenamiento asesoradas por profesores experimentados. & $\mathbf{3 , 7 4}$ \\
E3. Observaciones por supervisores, compañeros o grupos, y/o grabación en & 3,04 \\
vídeo de los profesores principiantes en las clases. & \\
F3. Entrevistas de seguimiento con observadores. & 3,08 \\
G3. Consultas a profesores con experiencia. & $\mathbf{4 , 0 3}$ \\
H3. Apoyo y asesoramiento sistemático pro profesores mentores. & $\mathbf{3 , 7 5}$ \\
I3. Oportunidad para observar a otros profesores. & $\mathbf{3 , 7 4}$ \\
J3. Reducción del tiempo/carga docente. & 2,69 \\
K3. Reuniones en grupo de profesores principiantes. & 3,30 \\
L3. Reuniones en grupo de profesores noveles asesorados por un experto. & 3,48 \\
M3. Creación de situaciones de enseñanza en equipo. & 3,42 \\
N3. Cursos específicos para profesores principiantes en la universidad. & 3,35 \\
O3. Publicaciones pedagógicas específicas para profesores principiantes. & 2,96
\end{tabular}

Tabla 9

Puntuación de la Dimensión 4: medidas para disminuir problemas del profesorado principiante

\begin{tabular}{lc}
\hline Ítems & Media \\
\hline A4. Elevar y mejorar la teoría en la formación inicial. & 3,15 \\
B4. Cualificar la formación permanente. & 3,66 \\
C4. Mejorar los sistemas de selección. & 3,77 \\
D4. Ampliar su autonomía profesional. & 3,70 \\
E4. Apoyarle en los conflictos con los padres. & $\mathbf{4 , 0 2}$ \\
F4. Respaldar su autoridad en los conflictos escolares en general. & $\mathbf{4 , 0 8}$ \\
G4. Mentorizar al profesor en los primeros años de su práctica docente. & 3,55 \\
H4. Clarificar ante la sociedad las funciones propias del profesorado, de las & 3,88 \\
\hline
\end{tabular}


familias y las que deben ser objeto de actuación conjunta.

I4. Dotarle de más medios para mejorar los resultados de sus alumnos. $\quad 3,80$

J4. Realizar actividades de formación de padres. 3,66

K4. Trabajar para aumentar la participación de la familia en el centro. 3,73

L4. Potenciar y cualificar las prácticas en la formación inicial. $\quad 3,88$

M4. Mayor formación en el conocimiento de las principales conductas $\quad \mathbf{4 , 0 6}$ disruptivas del alumnado

N4. Observación de profesores experimentados $\quad 3,86$

Tabla 10

Puntuación de la Dimensión 5: medidas para disminuir los problemas del profesorado con experiencia

\begin{tabular}{lc}
\hline Ítems & Media \\
\hline A5. Elevar y mejorar la teoría en la formación inicial & 3,15 \\
B5.Mejorar la formación permanente & 3,80 \\
C5. Mejorar los sistemas de selección & 3,66 \\
D5. Ampliar su autonomía profesional & 3,91 \\
E5. Apoyarle en los conflictos con los padres & $\mathbf{4 , 1 2}$ \\
F5. Respaldar su autoridad en los conflictos escolares & $\mathbf{4 , 2 6}$ \\
G5.Clarificar ante la sociedad las funciones propias del profesorado, de las & $\mathbf{4 , 1 1}$ \\
familias y las que deben ser objeto de actuación conjunta & $\mathbf{4 , 0 7}$ \\
H5. Dotar al profesorado de más medios para mejorar los resultados de sus & \\
alumnos & 3,93 \\
I5. Trabajar para aumentar la participación de la familia en el centro. & 3,66 \\
J5. Potenciar y cualificar las prácticas en la formación inicial & 3,87 \\
K5. Mayor formación en el conocimiento de las principales conductas & \\
disruptivas del alumnado & 3,96 \\
L5. Incentivar el intercambio de experiencia y buenas prácticas & \\
\hline
\end{tabular}


Tabla 11

Puntuación de la Dimensión 6: iniciativas para mejorar el desempeño docente

\begin{tabular}{lc}
\hline Ítems & Media \\
\hline A6. Facilitar una mayor formación del profesorado en metodología & 3,81 \\
didáctica. & 3,86 \\
B6. Potenciar la valoración de lo actitudinal y lo emocional en el alumnado & 3,98 \\
C6. Impulsar el desarrollo de estrategias de enseñanza-aprendizaje & 3,71 \\
D6. Sistematizar la reflexión en y sobre la práctica & 3,96 \\
E6. Profundizar en la calidad humana de las relaciones profesor-familia- \\
alumno & 3,83 \\
F6. Buscar un equilibrio armónico entre las funciones gestora y educativa en & \\
el centro y aula & 3,86 \\
G6. Incrementar el trabajo en equipo de los docentes & 3,82 \\
H6. Ampliar la presencia de educadores y trabajadores sociales en los & \\
centros & 3,91 \\
I6. Dotar de mayores recursos a los Servicios de Orientación & 4,24 \\
J6. Optimizar el uso de los recursos humanos, curriculares, culturales y \\
materiales del entorno
\end{tabular}

Si comparamos estos resultados según los distintos grupos creados, obtenemos los siguientes resultados:

\section{A. Según la edad de los docentes}

En las dimensiones 1, 2, 3 y 6 no hay diferencias significativas según la edad de los docentes, por lo que se obtienen los mismos resultados que en el apartado anterior.

En la Dimensión 4, los y las docentes menores de 35 años y los mayores de 57 años, eligen el ítem E4 Apoyarle en los conflictos con los padres. Los y las docentes entre 35 y 46 años, señalan el ítem F4 Respaldar su autoridad en los conflictos escolares en general como la medida más influyente en los problemas de los principiantes, mientras que los y las docentes de entre 46 y 57, eligen el M4 Mayor formación en el conocimiento de las principales conductas disruptivas del alumnado.

En la Dimensión 5, el ítem I5 Trabajar para aumentar la participación es más valorado por los menores de 35 años y el ítem F5 Respaldar su autoridad en los conflictos escolares es el elegido por todos los demás profesores.

\section{B. Según los años de experiencia docente}

Existen algunas diferencias entre los docentes con distintos años de experiencia. En la Dimensión 1, 2, 3, 5 y 6 todos los resultados no varían con respecto a los resultados generales, siendo éstas la Y, C2, G3, J5 y la J6. 
En la Dimensión 4, los principiantes eligen las subdimensiones L4 Potenciar y cualificar las prácticas en la formación inicial; los que tiene 5 y 15 años de profesión eligen la F4 Respaldar su autoridad en los conflictos escolares en general; los y las docentes que cuentan entre 15 y 20 años de experiencia eligen el H4 Clarificar ante la sociedad las funciones propias del profesorado, de las familias y las que deben ser objeto de actuación conjunta. Y los que más experiencia tienen prefieren la M4 Mayor formación en el conocimiento de las principales conductas disruptivas del alumno.

\section{Según el nivel educativo que imparten}

Teniendo en cuenta los niveles de enseñanza de los docentes, no hay diferencias con respecto a los resultados generales en las Dimensiones 2, 3 y 6 .

En la Dimensión 1 los resultados son los siguientes:

Tabla 12

Puntuación de la Dimensión 1: Problemas que afectan en el aula y en el centro según el nivel educativo

\begin{tabular}{|c|c|c|c|c|}
\hline Dimensión 1 & Infantil & Primaria & $\begin{array}{c}\text { ESO y } \\
\text { Bachillerato }\end{array}$ & $\begin{array}{l}\text { Formación } \\
\text { Profesional }\end{array}$ \\
\hline \multicolumn{5}{|c|}{ Puntuaciones Medias } \\
\hline $\mathbf{A}$ & 4,17 & 4,02 & 4,07 & 3,40 \\
\hline B & 4,29 & 3,98 & 4,09 & 4,25 \\
\hline $\mathbf{C}$ & 3,94 & 3,60 & 3,65 & 3,25 \\
\hline $\mathbf{D}$ & 2,47 & 3,22 & 3,23 & 2,95 \\
\hline $\mathbf{E}$ & 3,00 & 3,48 & 3,25 & 2,30 \\
\hline $\mathbf{F}$ & 3,29 & 3,60 & 3,49 & 3,75 \\
\hline G & 3,52 & 3,20 & 3,16 & 3,70 \\
\hline $\mathbf{H}$ & 4,00 & 3,30 & 3,18 & 3,10 \\
\hline $\mathbf{I}$ & 4,11 & 4,07 & 3,36 & 3,60 \\
\hline $\mathbf{J}$ & 2,05 & 2,95 & 2,70 & 2,95 \\
\hline $\mathbf{K}$ & 2,70 & 3,55 & 2,89 & 3,65 \\
\hline $\mathbf{L}$ & 2,64 & 3,05 & 2,85 & 2,95 \\
\hline $\mathbf{M}$ & 2,52 & 2,98 & 2,92 & 2,75 \\
\hline $\mathbf{N}$ & 2,88 & 3,30 & 3,01 & 3,25 \\
\hline $\mathbf{O}$ & 2,41 & 3,20 & 2,98 & 3,95 \\
\hline $\mathbf{P}$ & 3,05 & 3,69 & 3,30 & 3,35 \\
\hline $\mathbf{Q}$ & 2,41 & 3,02 & 2,87 & 3,35 \\
\hline $\mathbf{R}$ & 2,82 & 3,07 & 3,07 & 3,45 \\
\hline $\mathbf{S}$ & 3,23 & 3,54 & 3,29 & 2,95 \\
\hline $\mathbf{T}$ & 2,23 & 3,13 & 2,94 & 2,75 \\
\hline $\mathbf{U}$ & 1,70 & 2,86 & 2,69 & 2,85 \\
\hline $\mathbf{V}$ & 2,88 & 3,75 & 3,01 & 3,45 \\
\hline $\mathbf{X}$ & 3,76 & 3,52 & 2,85 & 3,15 \\
\hline $\mathbf{Y}$ & 4,70 & 4,39 & 4,05 & 4,00 \\
\hline
\end{tabular}




\begin{tabular}{lllll}
\hline $\mathbf{Z}$ & 3,35 & 3,20 & 3,25 & 3,65 \\
\hline
\end{tabular}

Tabla 13

Aspectos que interfieren en el desarrollo docente según el nivel educativo

\begin{tabular}{|c|c|c|c|c|}
\hline & Infantil & Primaria & $\begin{array}{l}\text { Secundaria y } \\
\text { Bachillerato }\end{array}$ & $\begin{array}{l}\text { Formación } \\
\text { Profesional }\end{array}$ \\
\hline $1^{o}$ & $\begin{array}{l}\text { Número de } \\
\text { alumnos por aula }\end{array}$ & $\begin{array}{l}\text { Número de } \\
\text { alumnos por } \\
\text { aula }\end{array}$ & $\begin{array}{l}\text { Motivación de los } \\
\text { alumnos }\end{array}$ & $\begin{array}{l}\text { Motivación de los } \\
\text { alumnos }\end{array}$ \\
\hline $2^{o}$ & $\begin{array}{l}\text { Motivación de } \\
\text { los alumnos }\end{array}$ & $\begin{array}{l}\text { Sobrecarga de } \\
\text { trabajo }\end{array}$ & Disciplina en el aula & $\begin{array}{l}\text { Número de alumnos } \\
\text { por aula }\end{array}$ \\
\hline $3^{\circ}$ & $\begin{array}{l}\text { Disciplina en el } \\
\text { aula }\end{array}$ & $\begin{array}{l}\text { Disciplina en el } \\
\text { aula }\end{array}$ & $\begin{array}{l}\text { Número de alumnos } \\
\text { por aula }\end{array}$ & $\begin{array}{c}\text { Dominio de las } \\
\text { materias }\end{array}$ \\
\hline $4^{\circ}$ & $\begin{array}{l}\text { Sobrecarga de } \\
\text { trabajo }\end{array}$ & $\begin{array}{l}\text { Motivación de } \\
\text { los alumnos }\end{array}$ & $\begin{array}{c}\text { Adaptación de la } \\
\text { enseñanza a las } \\
\text { diferencias individuales }\end{array}$ & $\begin{array}{l}\text { Organización del } \\
\text { trabajo en clase }\end{array}$ \\
\hline $5^{\circ}$ & $\begin{array}{l}\text { Problemas con } \\
\text { alumnos en } \\
\text { concreto }\end{array}$ & $\begin{array}{c}\text { Falta de tiempo } \\
\text { libre }\end{array}$ & $\begin{array}{l}\text { Organización del } \\
\text { trabajo en clase }\end{array}$ & $\begin{array}{l}\text { Insuficiencia de } \\
\text { material }\end{array}$ \\
\hline
\end{tabular}

En la Dimensión 4, todos los docentes, excepto los de Formación Profesional, eligen el ítem M4 Mayor formación en el conocimiento de las principales conductas disruptivas del alumnado, aquellos eligen el E4 Apoyarle en los conflictos con los padres y el F4 Respaldar su autoridad en los conflictos escolares en general.

En la Dimensión 5, los docentes de Infantil eligen el H5 Dotar al profesorado de más medios para mejorar los resultados de sus alumnos, los de Primaria, Secundaria y Bachillerato eligen el F5 Respaldar su autoridad en los conflictos escolares; mientras que los docentes de Formación Profesional eligen el F5 y, además, el G5 Clarificar ante la sociedad las funciones propias del profesorado, de las familias y las que deben ser objeto de actuación conjunta.

\section{Conclusiones/discusión}

Los aspectos que influyen en el desempeño de la función docente son la ratio, la motivación del alumnado, la disciplina en el aula, la sobrecarga de trabajo y la adaptación de la enseñanza a las diferencias individuales.

Sin embargo, estos aspectos difieren según el nivel educativo. El número de alumnos y alumnas parece tener más importancia para los y las docentes de Educación Infantil y Educación Primaria, mientras que para los y las profesionales de Secundaria y niveles postobligatorios es la motivación del alumnado el aspecto que más les afecta.

Dado que el nivel educativo y los años de experiencia influyen en el desempeño de la formación docente, se hace necesario apoyar a los docentes noveles con tutorización de otros profesionales o formación permanente, así como con estrategias de colaboración en aquellos niveles educativos que presentan más problemática.

Uno de los resultados, cuanto menos curioso, es la poca influencia que ejerce el tratamiento 
de la interculturalidad en las aulas, teniendo en cuenta que este estudio se lleva a cabo en la ciudad de Melilla y se caracteriza precisamente por la convivencia de varias culturas. Esta falta de influencia en el desempeño docente tendría que ser investigada para averiguar la causa, ya que no se puede decir si es un aspecto que está totalmente manejado por el profesorado o si simplemente no se tiene en cuenta como tema transversal a la hora de desarrollar la docencia.

En cuanto a la identificación de las estrategias conducentes a la mejora del desempeño de la función docente, se establecen las siguientes conclusiones:

La mejora del uso de los recursos, tanto humanos, contextuales y materiales como culturales y curriculares, y la conexión entre el currículo y el entorno, podría ser una necesidad del sistema educativo actual, hecho que podría dar origen a metodologías recientes como las Comunidades de Aprendizaje.

Por otra parte, el desarrollo de estrategias de enseñanza-aprendizaje por el que opta, podría relacionarse con la innovación metodológica por la que se insta desde congresos, investigaciones y foros educativos.

Los y las docentes, además, seleccionan la profundización en la calidad humana de las relaciones profesorado-familia y alumnado, siendo éste un ámbito que aún hoy día se trabaja en defecto desde la escuela y que sigue tratándose de la misma manera que desde hace años: a través de la Asociación de Padres y Madres y de las tutorías. Hacer más partícipes a los padres y madres del alumnado en el proceso educativo de sus hijos e hijas, debe pasar por hacerles conscientes de la importancia que tiene y otorgarles un papel activo en las diferentes decisiones. Por otra parte, dotar de mayores recursos a los servicios de Orientación es una muestra de lo necesario que resulta este agente para el resto de docentes, ya que actúan de intermediarios entre el alumnado y la realidad, entre los y las docentes y discentes y entre la familia y la escuela.

Con respecto a la identificación de los rasgos del profesorado principiante y las estrategias para solventar sus dificultades, se puede concluir que:

Los y las docentes con poca experiencia laboral se caracterizan por el estrés y la angustia que viven en situaciones complicadas y la necesidad de mucha más energía, tiempo y concentración para resolver los problemas que el profesorado con experiencia. Estos resultados coindicen con estudios anteriores como el de García (2000), donde se establece que la organización y control son dos aspectos clave. Además, necesitan un apoyo extra durante sus primeros años de trabajo, coincidiendo con las ideas de Tiana (2013).

Con respecto a las mejores estrategias para que los programas de iniciación a la docencia tengan éxito, se resumen las siguientes ideas:

- Consultas a profesores con experiencia.

- Apoyo y asesoramiento sistemático por profesores mentores.

- Oportunidad para observar a otros profesores.

- Sesiones de entrenamiento asesoradas por profesores experimentados.

La utilización de estas estrategias, podrían ser valoradas para comprobar las competencias del profesorado novel (Regan, Anctil, Dubea, Hofmann y Vaillancourt, 2002) y comprobar la eficacia de las mismas.

Por otro lado, el respaldo de la autoridad docente es una necesidad tanto para docentes expertos como para principiantes, pero estos últimos necesitan mayor formación en el conocimiento de las principales conductas disruptivas del alumno. Los propios docentes sin experiencia establecen que aumentar las prácticas en la formación inicial e intercambiar experiencias serían las medidas más 
apropiadas.

Con respecto a las medidas para evitar o disminuir los problemas del profesorado experto, difieren de las de sus colegas con menos experiencia. La dilucidación ante la sociedad de las funciones propias del profesorado y de las familias parece ser la medida más valorada para mejorar su situación laboral, debiéndose quizá a la pérdida de autoridad que ha vivido el y la profesional de la educación con respecto a épocas anteriores.

Finalmente, este estudio deja a la luz la importancia de una formación académica en coherencia con la realidad educativa, en contacto con las aulas y con la colaboración de profesores experimentados, que desarrolle oportunidades de role-playing y de observación de expertos, de tal forma que la pedagogía y la didáctica formen parte de la preparación del docente. (Arias y Jiménez, 2013).

\section{Referencias}

Arias, W.L. y Jiménez, N.A (2013). Síndrome de burnout en docentes de Educación Básica Regular de Arequipa. Educación, 22(42), 53-76.

Arteaga, B. y García, M. (2008). La formación de competencias docentes para incorporar estrategias adaptativas en el aula. Revista Complutense de Educación, 19(2), 253-274.

Cardona, J. (2008a). Formación y desarrollo profesional del docente en la sociedad del conocimiento. Madrid: Universitas.

Cardona, J. (2008b). Cuestiones en torno a la formación y desarrollo profesional de los profesores. Madrid: Sanz y Torres.

Castejón , J.L. (2010). Diferencias de género y curso en metas académicas en alumnos de Educación Secundaria Obligatoria. Revista española de pedagogía, 68(245), 67-84.

Diker, G. y Terigi, F. (1997). La formación de maestros y profesores: hoja de ruta. Buenos Aires: Paidós.

Epelde, A. (2012). Problemática de los alumnos de magisterio durante el prácticum: Estudio en la Facultad de Educación y Humanidades de Melilla. Publicaciones de la Facultad de Educación y Humanidades del Campus de Melilla, (42), 107-120.

Feliz, T.M, y Ricoy, M.C. (2008). La formación inicial de los maestros. En J. Cardona, Cuestiones en torno a la formación y desarrollo profesional de los profesores, (pp. 3-38). Madrid: Sanz y Torres.

García-Cabero, M. (2000). Proyecto docente de la asignatura Psicología de la Instrucción. León: Universidad de León.

Gil, J.A. (2011). Metodología cuantitativa en educación. Madrid: UNED.

González, B. (1999). Los estereotipos como factor de socialización en el género. Comunicar, 12, 7988.

Imbernón (1998). La formación y el desarrollo profesional del profesorado. España: Biblioteca de Aula.

Infante, M. (2010). Desafíos a la formación docente: inclusión educativa. Estudios Pedagógicos, 36(1), 287-297.

López, E. y Pérez, E. (2013). Formación permanente del profesorado y práctica docente intercultural: contenidos actitudinales y complementariedad competencial. Espiral. Cuadernos del Profesorado, 6(12), 32-42. Recuperado de http://www.cepcuevasolula.es/espiral

Márquez, A.C. (2009). La formación inicial para el nuevo perfil del docente de Secundaria. Relación entre la teoría y la práctica. (Tesis Doctoral). Universidad de Málaga, Málaga, España.

Mckinsey \& Company (2007). Women Matter. Gender diversity, a corporate performance driver. Mckinsey \& 
Company.

Medina, A. (Coord.) (2015). Innovación de la educación y de la docencia. Madrid: Ramón Areces.

Mérida, R. (2009). Necesidades actuales en la formación inicial de las maestras y maestros. Revista electrónica interuniversitaria de formación del profesorado, 12(2), 39-47.

Moral, C. (2000). Formación para la profesión docente. Revista Interuniversitaria de Formación del Profesorado, 37, 171-186.

Osorio, J. A., Herrer, L. y Quiles, O. (2016). Competencias docentes de los profesores en formación según opinión de los docentes formadores. Diálogos, 17, 35-51.

Pérez, M.S.T. (2016). An exploratory inquiry study of Guam teachers' perceptions in managing disruptive behaviors. (Tesis Doctoral). Universidad de Fénix, Arizona. Recuperado de https://search.proquest.com/openview/c402333ec4a64bab95e7bfb93252b8e4/1?pqorigsite $=$ gscholar\&cbl $=18750 \&$ diss $=\mathrm{y}$

Ramírez, S., Sánchez, C.A., García, A. y Latorre, M.J. (2011). El Prácticum en Educación Infantil, Primaria y Máster de Secundaria. REDU: Revista de Docencia Universitaria, 9(2), 257-259.

Rebolledo, T. (2015). La formación inicial del profesorado de educación primaria y Secundaria en Alemania, España, Finlandia Francia y Reino Unido. Estudio comparado. Revista Española de Educación Comparada, 25, 129-148.

Regan, H.B., Anctil, M., Dubea, C., Hofmann, J. y Vaillancourt, R. (2002). El profesor una nueva definición... y un nuevo modelo de evaluación y actualización profesional. Madrid: Editorial Centro de Estudios Ramón Areces.

Rico, A.M. (2006). La importancia de la práctica docente en la didáctica de la Lengua Española como asignatura troncal de los estudios de Magisterio. Revista Electrónica Interuniversitaria de Formación del Profesorado, 9(1), 1-18.

Rojas, N.; Carrillo, J. y Flores, P. (2012). Características para identificar a profesores de matemáticas expertos. En A. Estepa, A. Contreras, J. Deulofeu, M.C. Penalva, F.J. García, y L. Ordónez, (Eds.), Investigación en Educación Matemática XVI, (pp. 479-485). Jaén: Sociedad Española de Investigación en Educación Matemática.

SATE (2013). Riesgo de fragmentación social en Melilla y Ceuta. Revista Escuela Pública, (57).

Tiana, A. (2013). Los cambios recientes en la formación inicial del profesorado en España: una reforma incompleta. Revista Española de Educación Comparada, 22, 39-58.

Rodríguez, H. y Torrego, L. (2013). Educación inclusiva, equidady derecho a la diferencia: transformando la escuela. Madrid: Wolters Kluwer España

Valverde, J., Garrido, M.C. y Fernández, R. (2010). Enseñar y aprender con tecnologías: un modelo teórico para las buenas prácticas con TIC. Teoría de la Educación. Educación y Cultura en la Sociedad de la Información, 11(1), 203-229.

Vázquez, G., Sarramona, J. y Touriñan, J. M. (2010). La escuela en crisis. En J. V. Peña y Mª C, Fernández (Coords.), La escuela en crisis, (pp. 17-78). Barcelona: Octaedro.

Veenman, S. (1984). Perceived problems of beginning teachers. En J. Cardona, (2008b), Cuestiones en torno a la formación y desarrollo profesional de los profesores. Madrid: Sanz y Torres.

\section{Información sobre los autores}

Autor: Irene Orellana Román

Institución: Universidad de Granada

Email: irene1986es@gmail.com 


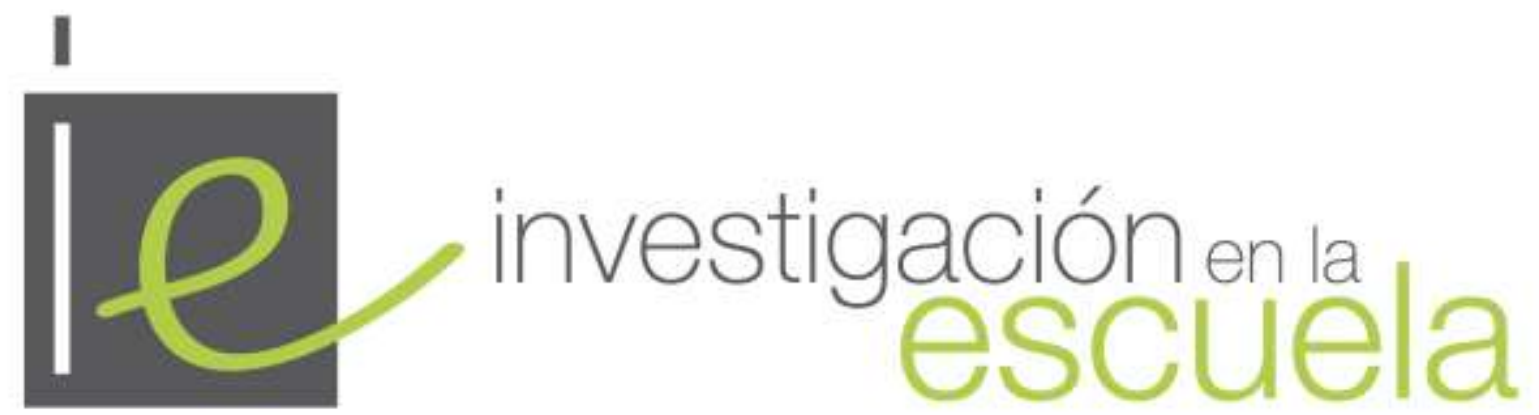

Revista académica evaluada por pares y de acceso abierto

Número 95

15 de octubre de 2018

ISSN 2443-9991

\section{@}

SOMIERIGHISRESERVEDLOS/as lectores/as pueden copiar, mostrar, y distribuir este artículo, siempre y cuando se de crédito y atribución al autor/es y a Investigación en la Escuela, se distribuya con propósitos no-comerciales, no se altere o transforme el trabajo original. Más detalles de la licencia de CreativeCommons se encuentran en http://creativecommons.org/licenses/by-nc-sa/3.0 Cualquier otro uso debe ser aprobado en conjunto por el autor/es, o Investigación en la Escuela.

ư

Revista Editada por la Universidad de Sevilla. https://editorial.us.es/es/revistainvestigacion-en-la-escuela

Contribuya con comentarios y sugerencias en la web de la revista. Por errores y sugerencias contacte a secretaria@investigacionenlaescuela.es 


\section{Investigación en la escuela}

Consejo de dirección: Ana Rivero García (Universidad de Sevilla), Nicolás de Alba Fernández (Universidad de Sevilla), Pedro Cañal de León (Universidad de Sevilla), Francisco F. García Pérez (Universidad de Sevilla), Gabriel Travé González (Universidad de Huelva), Francisco F. Pozuelos Estrada (Universidad de Huelva)

Dirección: Ana Rivero García y Nicolás de Alba Fernández

Secretaría de edición: Elisa Navarro Medina

\section{Consejo editorial}

José Félix Angulo Rasco. Universidad de Cádiz Rosa Ma Ávila Ruiz. Universidad de Sevilla Pilar AzcárateGoded. Universidad de Cádiz Juan Bautista Martínez Rodríguez. Universidad de Granada

Nieves Blanco García. Universidad de Málaga Fernando Barragán Medero. Universidad de La Laguna José Carrillo Yáñez. Universidad de Huelva José Contreras Domingo. Universidad de Barcelona. Luis C. Contreras González. Universidad de Huelva Ana $\mathbf{M}^{\mathbf{a}}$ Criado García-Legaz. Universidad de Sevilla Rosario Cubero Pérez. Universidad de Sevilla José Ma Cuenca López. Universidad de Huelva Jesús Estepa Giménez. Universidad de Huelva Rafael Feito Alonso. Universidad Complutense (Madrid)

Francisco José García Gallardo. Universidad de Huelva

Soledad García Gómez. Universidad de Sevilla J. Eduardo García Díaz. Universidad de Sevilla
Fernando Hernández Hernández. Universidad de Barcelona

Salvador Llinares Ciscar. Universidad de Alicante Alfonso Luque Lozano. Universidad de Sevilla Rosa Martín del Pozo. Universidad Complutense (Madrid)

José Martín Toscano. IES Fernando Herrera (Sevilla) Jaume Martínez Bonafé. Universidad de Valencia F. Javier Merchán Iglesias. Universidad de Sevilla Emilia Moreno Sánchez. Universidad de Huelva. Rosario Ortega Ruiz. Universidad de Córdoba Antonio de Pro Bueno. Universidad de Murcia Fco. de Paula Rodríguez Miranda. Universidad de Huelva

Pedro Sáenz-López Buñuel. Universidad de Huelva Antoni Santisteban Fernández. Universidad Autónoma (Barcelona)

Emilio Solís Ramírez. Catedrático de IES. $\mathbf{M}^{\mathbf{a}}$ Victoria Sánchez García. Universidad de Sevilla. Magdalena Suárez Ortega. Universidad de Sevilla

\section{Consejo asesor}

Manuel Área Moreira. Universidad de La Laguna Jaume Carbonell. Director Cuadernos de Pedagogía. Barcelona César Coll. Universidad de Barcelona

Christopher Day. Universidad de Nothingham. U.K.

Juan Delval. Universidad Nacional de Educación a Distancia

John Elliott. Universidad de East Anglia. Norwich. U.K.

José Gimeno Sacristán. Universidad de Valencia

André Giordan. Universidad de Paris VII y Ginebra

Francisco Imbernón. Universidad de Barcelona Ángel Pérez Gómez. Universidad de Málaga

Rafael Porlán Ariza. Universidad de Sevilla

Francesco Tonucci. Instituto de Pedagogía del C.N.R. Roma

Jurjo Torres Santomé. Universidad de A Coruña 
